\title{
A CFD-PBM coupled model under entire turbulent spectrum for simulating a bubble column with highly viscous media
}

\author{
Huahai Zhang ${ }^{1}$, Yuelin Wang ${ }^{1}$, Ali Sayyar ${ }^{1}$, and Tiefeng Wang ${ }^{1}$ \\ ${ }^{1}$ Tsinghua University
}

April 28, 2021

\begin{abstract}
To account for the effect of liquid viscosity, the bubble breakup model considering turbulent eddy collision based on the inertial subrange turbulent spectrum was extended to the entire turbulent spectrum that included the energy-containing, inertial, and energy-dissipation subranges. The computational fluid dynamics-population balance model (CFD-PBM) coupled model was modified to include this extended bubble breakup model for simulations of a bubble column. The effect of turbulent energy spectrum on the bubble breakup and hydrodynamic behaviors was studied in a bubble column under different liquid viscosities. The results showed that when the liquid viscosity was $<80 \mathrm{mPa}$ ?s, the bubble breakup and hydrodynamics were almost independent on the turbulent spectrum. At liquid viscosity $>80 \mathrm{mPa}$ ?s, the bubble breakup rate and gas holdup were significantly under-predicted when the inertial turbulent spectrum was used, and when using the entire turbulent spectrum the predictions were more consistent with experimental data.
\end{abstract}

\section{Hosted file}

Entire Spectrum_20210413.pdf available at https://authorea.com/users/404261/articles/520008a-cfd-pbm-coupled-model-under-entire-turbulent-spectrum-for-simulating-a-bubble-columnwith-highly-viscous-media 